Guido Wolf

OBER SEMANTISCHE VERKORZUNGEN IN ALLTAGSGESPRACHEN

Zur Applikation eines rahmentheoretischen Konzepts

auf empirisches Material

0. Einleitung

1. Ein kommunikations theoretischer Frame-Ansatz

2. Semantische Verkürzung: Ein Beispiel

2.1. Frame-theoretische Deskription

2.2. Zur Interpretation der Gesprächssequenz

\title{
0. Einleitung
}

Alltägliche Gespräche sind häufig durchsetzt mit darstellend-erzählenden Passagen, die unterschiedliche Funktionen haben können. Ist ihre Funktion in der konkreten Situation nach Meinung der Sprechenden, gemessen an gesetzten Zielen und Zwecken, erfüllt, ${ }^{1)}$ so werden diese Passagen beendet, ohne weitere Details mitzuteilen. Manchmal jedoch sind Erzählungen über ihre prinzipielle Unvollständigkeit hinaus von semantischer Verkürzung gekennzeichnet, so etwa in Fällen, in denen die Sprechenden persönliche Ziele wie den Schutz der eigenen Intimsphäre verfolgen. Diese Intention verletzt jedoch unter Umständen den berechtigten Anspruch der Hörer auf ausreichende Explikation.

Im folgenden möchte ich anhand der Analyse eines konkreten Beispiels eine spezifische Lösungsmöglichkeit für einen solchen Fall darstellen. Als Analyseinstrument dient ein kommunikationstheoretisch ausgelegter Frame-Ansatz, den ich im ersten Abschnitt entfalte.

1. Ein kommunikationstheoretischer Frame-Ansatz

Seit den siebziger Jahren finden ursprünglich kognitionspsychologische Konzepte, die menschliche Denkoperationen als schematisch antizipierend und zielorientiert beschreiben, ${ }^{2)}$ verstärkt in mit der Erforschung menschlicher Kommunikation im weitesten Sinn befaßten Wissenschaften Beruicksichtigung. In 
jüngster Zeit legte Müller (1984) für die Gesprächsanalyse eine "Rahmenanalyse des Dialogs" vor, die mittels eines frame-theoretischen Ansatzes zahlreiche Aspekte sprachlicher Verständigung in Alltagsgesprächen herausarbeitet.

Gerade die Heterogenität der wissenschaftlichen Anknüpfungen an den frametheoretischen Grundgedanken, daß nämlich Sinnesdaten strukturiert wahrgenommen und in bestehende Schemata von Erfahrung eingeordnet werden, führte zu einer Vielzahl weitgehend synonymer Ternini: Statt von "Frames" sprechen verschiedene Autoren von "Scripts", "Plans", "Schemata", "Rahmen" etc. Im folgenden wird der Ausdruck Frame 3 ) verwendet, der eine nach spezifischen Kriterien geordnete, kognitive Datenstruktur bezeichne, die inhaltliches und operationales Wissen enthält und eingebettet ist in die je "individuelle Welttheorie". 4)

Frames ennöglichen die Identifizierung einer konkreten Erfahrung qua schematisierender Einordnung und liefern Handlungsmuster für den Umgang mit dieser Erfahrung. Sie sind jedoch nicht allein statische und individuelle Entitäten, sondern auch prozedural-dynamische und sozial deteminierte, potentiell fragile und permeable Konstrukte, die veränderbar sind, wenn es die Situation erfordert. Sie überschneiden sich und können zu hierarchisch konstituierten Frame-Systemen gebuindelt werden. Es ergibt sich die verwirrende, aber nicht aufhebbare Konsequenz, daß Frames als strukturierende Klassifikatoren benutzt werden, ihrerseits aber offen, unscharf und durch konkrete Erfahrung modifizierbar sind. Ihre Funktion erfüllen sie jedoch nicht nur für Wahrnehmungsprozesse, sondern auch in bezug auf die Planung und Ausführung von Handlungen - und somit für das Hören und Verstehen wie für das Sprechen. ${ }^{5)}$

Gespräche können also beschrieben werden als Frame-basierte Handlungen, in denen Teilnehmer von jeweils adäquaten Frames der äußeren Situation, der Beteiligten und der Gesprächsthemen ausgehen. Der Verlauf eines Gesprächs erfordert jedoch möglicherweise nicht nur Modifikationen einzelner applizierter Frames. Es können im situativen Progreß neue Frames entstehen (z.B. Wissen über vorher Gesagtes, über Intentionen der anderen etc.), die den Set erweitern. ${ }^{6)}$ Eine konkrete Außerung wird also produziert bzw. verstanden im Schnittpunkt eines je individuell aktualisierten Sets von Frames. Verständigung zwischen Kommunikationspartnern ließe sich dann beschreiben als Koordination dieser Sets. Der Bezug auf Frames wird kommunikativ indiziert und damit des Sprechers Appelle an den Hörer, seinerseits Frames zu aktualisieren. Der Sprecher setzt diese zumindest zum Teil als gleichartig seinen eigenen gegenüber voraus. Ich konzentriere mich im folgenden auf den semantischen Aspekt von Gesprächen, wobei deren Eingebundensein in soziale Situationen nicht aus dem Blick geraten soll. 7) 
Als Bezeichnung für die kognitiven semantischen Repräsentationen zum generellen Gegenstand eines Gesprächs schlage ich den Ausdruck Topical-Frame vor. Hierin eingebettet sind Macro-Frames, thenatische Aspekte des TopicalFrame, die noch auf einem "Global-Level" (van Dijk) liegen. Diese globale Ebene sei dann gegeben, wenn eine Außerung entweder keinem Topical-Frame zuzuordnen ist (und somit selbst einen Topical-Frame konstituiert) oder lediglich einen Topical-Frame untergeordnet ist. Die Folge von Sätzen, Phrasen, Wörtern oder anderen Zeichenkörpern, die innerhalb einer kommunikativen Außerung realisiert werden, konstituieren auf den "Local-Level" (van Dijk) Propositional-Frames. Innerhalb einer Außerung kann die Aufmerksamkeitsausrichtung bzw. der Fokus auf einer Ebene oder zwischen verschiedenen Ebenen verlagert werden. Kommunikative Elemente, die einen solchen Fokuswechsel anzeigen, nenne ich Focus-Change-Marker. ${ }^{8)}$

Gespräche können unterschiedlich strukturiert werden. Hier relevant sind argumentative Struktur und narrative Struktur. Van Dijk spricht in diesem Zusammenhang von "superstructures". Da aber argumentative oder narrative Verknüpfungen von Frames auf den unterschiedlichen Ebenen ebenfalls als Rahmen (Metarahmen) wirken, seien sie als Super-Fromes bezeichnet, angesiedelt auf einen "Abstract-Level". 9) Sie sind in hohem Maße konventional isiert.

Tritt ein Sprecher in eine Argumentation bzw. eine Erzählung ein, so gelten für ihn aufgrund der konventionalisierten Super-Frames gewisse Anforderungen. In Anschluß an Kallmeyer/Schütze (1977) kann in Hinsicht auf die Darstellung von Sachverhalten in Kommunikation festgehalten werden, daß für die Sprecher drei "grundlegende Regeln beziehungsweise 'Zugzwänge' bestehen" (Kallmeyer/Schütze 1977, 162): der "Kondensierungszwang", der besagt, daß für eine überschaubare Darstellung irrelevante Einzelphänomene weggelassen bzw. zusammengefaßt werden müssen, der "Detaillierungszwang", der für eine adäquate Darstellung die ausreichende Explikation von "konstitutiven Elementen und ihre( $n$ ) Beziehungen zueinander" (a.a.0.) vorschreibt sowie der "Gestaltschließungszwang", aufgrund dessen die Darstellung des jeweiligen Sachverhalts gegenüber anderen Sachverhalten abgegrenzt werden muß. Es läßt sich also konstatieren: Beginnen Sprechende eine Außerung, so gehen sie bestimmte Verpflichtungen hinsichtlich der Ausgestaltung ihrer Außerung gegenüber den Hörern ein.

Damit sei jedoch nicht einen kruden Deterninismus das Wort geredet. Sprechende haben mannigfaltige Moglichkeiten, aus der Vielzahl an Frames Alternativen zu wählen. Die Entscheidung für die Aktualisierung eines bestimmten Sets von Frames wird getroffen in Hinsicht auf die optimale Erreichung der Ziele und Zwecke der Kommunikation. Das gilt für die Produktion von Außerungen 
wie für deren Verstehen durch den Hörer. Solches Handeln wird geneinhin als "strategisches Handeln" angesehen. ${ }^{10)}$ In Anlehnung an die mathematische Spieltheorie erfährt der Begriff der Strategie eine sehr weite Ausdehnung. Sowohl bei der Sprachproduktion wie beim Sprachverstehen werden in diesem Sinn Strategien auf das geneinsame Ziel der Verständigung hin verfolgt. Der Strategiebegriff soll hier allerdings nicht näher erörtert werden. Für diese Untersuchung sind lediglich solche fälle strategischen Handelns relevant, in denen ein Sprecher Zwecke mit seiner Außerung erreichen will, die das Ziel optimaler Verständigung relativieren. Das ist etwa der Fall, wenn der Sprecher besondere Probleme, die durch eine optimale Außerung auftreten könnten, antizipiert bzw. auf bereits entstandene Probleme reagiert. Strategisches Handeln ist in diesem Sinn also erforderlich, wenn der Sprecher Grund zu der Befürchtung hat, daß der Hörer diese Zwecke nicht zu akzeptieren bereit ist. In solchen Situationen müssen die Sprechenden kommunikative Züge auswählen, die das Einklagen der genannten Zugzwänge durch den Hörer möglichst ausschließen. Für Außerungen, die eine solche Strategie einleiten, schlage ich die Bezeichnung "Strategic-Move" vor. Besonders in Situationen, wo ein ausreichendes Befolgen des Detaillierungszwanges den Sprecher dazu brächte, ein für ihn zu hohes $\mathrm{Ma} B$ an Infornationen über seine inneren Einstellungen, Wünsche, Angste und Gefuihle preiszugeben, werden solche Strategien verfolgt. ${ }^{11)}$ Das möchte ich nun anhand eines transkribierten Gesprächsausschnittes denonstrieren.

\section{Semantische Verkürzung: Ein Beispiel}

Der hier untersuchte Gesprächsausschnitt entstanmt einem Korpus aus dem Forschungsprojekt "Traumerzählungen und Traumberichte in Alltagsgesprächen", durchgeführt am Institut für Kommunikationsforschung und Phonetik Bonn. 12) Sechs Teilnehmer und ein teilnehmender Beobachter führen Gespräche über ihre Träume, Traumgewohnheiten und Interpretationen und werden dabei auf Videoband aufgenommen. Die Aufnahmen werden sodann transkribiert. ${ }^{13)}$ Das nun zu analysierende Transkriptstück (das sich im Anhang findet) gehört zum ersten der bisher neun Gespräche. Einige Teilnehmer kennen sich von einen Gespräch über Träume, das vor ca. zwei Jahren im Rahmen eines Vorprojektes geführt wurde. Zwei Teilnehmende sind zum ersten Mal dabei, eine davon ist Cassis (C), deren Außerung hier analysiert wird.

\subsection{Frame- theoretische Deskription}

Als das Gespräch ca. 15 Minuten andauert, wird die Frage, ob man farbig oder schwarz-weiß träume, angeschnitten. Cassis überniment nach einigen Minuten den 
Turn, um einen bestimmten Aspekt beizutragen. Unter Applikation des vorgeschlagenen Modells komme ich zu folgender Deskription (vgl. unten die schematische Darstellung).

Topica]-Frame ist "Gespräch über Träume", hierin eingebracht und eingebunden der Macro-Frame "Farbempfindungen in Träumen". Cassis' Beitrag knüpft dort an durch das (Und) sowie das anaphorische das in Zeile 1. Ihr nun aktualisierter Propositional-Frame verbindet Farbe und wichtig, deren Abhängigkeit sie in Zeile 3 und 4 spezifiziert: Sachen oder irgendwelche symbole oder irgendusas wirden manchmal auch erst wichtig durch die Farbe. Diese zentrale Aussage illustriert sie nun durch eine eingeschobene narrative Passage, einleitend markiert durch das Jetat in Zeile 5. Die narrative Passage gliedert sich, beschrieben in den Termen van Dijks (1980), wie folgt: Episode 1 besteht aus dem Setting vor'n paar Tagen geträunt und dem Gegenstand des Traumes (sehr großer, sehr grïner Frosch irgendwie, Zeile 5 u. 6). Cassis wird von zwei Hörerinnen gestört (besonders durch Moet's Lachen, Zeile 7) und kommentiert deshalb kurz (Zeile 10, leider nicht verständlich), um den Turn zu behalten und die aufkommende Heiterkeit zu unterbinden (unten werde ich einen Beleg für diese Interpretation liefern). Sie tritt mit dem der in Zeile 10 wieder in die Traumerzählung ein, doch wechselt sie nun das Setting und beginnt, eingeleitet durch den Focus-Change-Marker wie in Zeile 10, Episode 2. Hier bildet Experiment das Setting, spezifiziert als neurophysiologisches Experiment, bei den Reizübertragungen in Muskeln untersucht werden. Beendet wird diese Episode, die als Traumassoziation anzusehen ist, durch den Unschärfeindikator oder was (Zeile 17). ${ }^{14)}$ Cassis tritt nun wieder, nach einer sehr kurzen Pause (im Transkript durch das Komma signalisiert), die zusammen mit den Tempuswechsel als Focus-Change-Marker fungiert, in Episode 3 ein, indem sie die Mitteilung des Ereignisses, anknüpfend an das der in Zeile 10, beendet. Ihre Evaluation (ganz) fürchterlich bezieht sich wahrscheinlich auf die gesamte bisherige Erzählung. Diese nenne ich narrative Passage 1.

Durch das im nachhinein dann aufgefalalso e $i n$ gefallen (Zeile 18 u. 19) wird die narrative Passage 2 eingeleitet: Cassis erzählt nun über ihre Interpretation des Traumes und ihre Assoziation. Der Wechsel in diese Passage wird außerdem durch den Tempuswechsel (Perfekt und Präsens, Zeile 18-23) sowie ihre Korrektur in Zeile 19 indiziert: auffalzen würde eher zu der Erlebnisperspektive des Traumes passen, $e i n$ fallen scheint besser dem Rahmen interpretierender Reflexion zu entsprechen.

Aus der narrativen Passage 2 wechselt Cassis nun noch einmal zurück in die Traumerzählung, wobei die Pause, das Aber als Focus-Change-Marker sowie der 
Tempuswechsel den Ubergang markieren (Zeile 23 und 24). Schon in Zeile 24 beendet sie endgültig die Traumerzählung, inden sie wiederum einen Tenpuswechsel in das Präsens vornimmt und auf ihr Erinnerungsvemögen eingeht. Die Benerkung über das Aufschreiben bezieht sich auf einen noch vor der Initiation des Macro-Frames "Farbenpfindungen in Träumen" behandel ten Aspekt des TopicalFrame und wird hier vernachlässigt.

Cassis leitet in Zeile 27, vollendet ab Zeile 30, einen neuen Macro-Frame ein: "Musikempfindungen in Träumen - Träume sind wie Filme", markiert durch das auch und einen erneuten Tempuswechsel in Zeile 27. Das also in derselben Zeile eröffnet jedoch eine Passage, die noch einmal Bezüge zu ihrer narrativen Passage 1 herstellt. Dieser als "ergänzender Nachschub" (Kallmeyer) fungierende Passus wird mit der kurzen Pause in Zeile 30 beendet, und Cassis aktualisiert nun endgïltig den neuen Macro-Frame. Sie berichtet (im Transkript ausgelassen) von zwei Träumen, die wie Filme von Musik begleitet waren und die sie als nächtliche Vorstellung empfand. Nach einer längeren Pause geht sie auf ihre Motivation ein, in eine privat organisierte Traumerzählungsgruppe einzutreten, die sich mittlerweile aufgelöst hat. Es ist, wie sie sagt, ihre Neugier auf die Traumgeschichten, nicht jedoch das Interesse am "psychologischen Auseinanderklambüsern". Nach einer erneuten längeren Pause greift eine andere Gesprächsteilnehmerin den Macro-Frame "Akustische Empfindungen in Träumen" auf.

\subsection{Zur Interpretation der Gesprächssequenz}

Cassis' Beitrag ist bis Zeile 23 anzusehen als Explikation ihres Propositional-Frame: "Trauminhalte werden wichtig durch die Farbe" (Zeile 1-4). Sie illustriert diese Aussage durch die narrative Passage 1. Aber erst mit der zweiten narrativen Passage, also der reflektierenden Interpretation in,bezug auf die Alltagswirklichkeit, wird der Gedankenbogen des aktualisierten Propositional-Frame abgeschlossen. Das Attribut wichtig kann nur innerhalb einer solchen Interpretation einem Trauminhalt attestiert werden. Daraus läßt sich auch begründen, weshalb die fragmentarische Traumerzählung mit der Experiment-Assoziation zur narrativen Passage 1 zusammengefaßt werden kann. Nur im Zusammenhang mit der Assoziation erhält der Traum die Erlebnisqualität, die dann ihrerseits erst den Bezug auf den spezifischen Ausschnitt von Cassis' All tagswirklichkeit zuläßt.

Damit wird die narrative Passage 2 für die Explikation des PropositionalFrame zur zentralen Stelle. Und genau diese Stelle, die erst näheren Aufschluß über die besondere Wichtigkeit der narrativen Passage 1 geben kann, ist 
Schematische Darstellung der Frame-theoretischen Interpretation

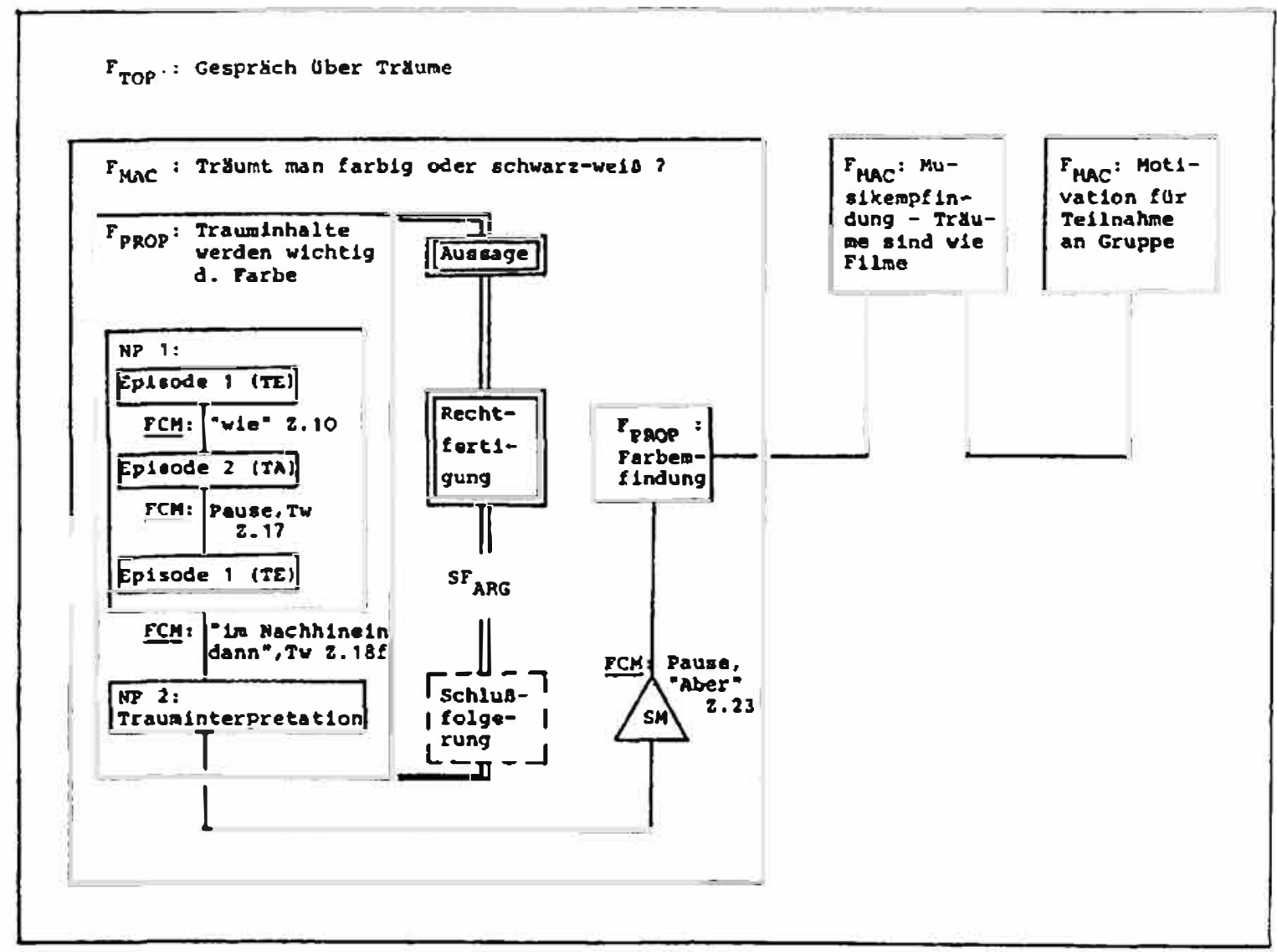

Zeichenerklärung:

$\mathrm{F}_{\text {TOP }}$ : Topical-Frame

$F_{\text {MAC : Macro-Frame }}$

$F_{\text {PROP }}$ : Propositional-Frame

$\mathrm{SF}_{\mathrm{ARG}}$ : argumentativer Super-Frame

NP 1/2: narrative Passage 1/2

TE : Traumerzählung

TA : Traumassoziation

FCM : Focus Change Marker

SM : Strategic Move

Z... : Zeile...

Tw: Tenpuswechsel

auf zwei Ebenen semantisch verkuirzt: den Local-Level und dem Abstract-Level. Zunächst zu letzterem.

Ich interpretiere Cassis' Außerungen bis $z u$ der Pause in Zeile 23 mit Hilfe eines argumentativen Super-Frames (siehe van Dijk 1980, 144ff.). Cass is trifft eine Aussage (den Propositional-Frame), die sie anschließend mit der Erzählung 
einer konkreten Erfahrung zu rechtfertigen sucht. Paraphrasiert werden könnte dieser Obergang von der Ausgangsbehauptung in die Kategorie "Rechtfertigung" so: "Ich kann das behaupten, weil ich so etwas schon erlebt habe." Beide narrative Passagen explizieren nun dieses Erlebnis, aber was fehlt, ist der explizite Rückbezug auf den Propositional-Frame. Damit bleibt die Kategorie "Schlußfolgerung" semantisch nicht ausgefuiltt (in der schematischen Darstellung durch die gestrichelte Linie um "Schlußfolgerung" visualisiert), Cassis äußert nichts, was ihre beiden narrativen Passagen auf das wichtig rückbezieht.

Auf dem Local-Level bleibt die narrative Passage 2 ebenfalls semantisch verkürzt. Weder wird Näheres über eine bestinente Ferson gesagt, noch wird die Metapher Versuchskaninchen, die ja an die Experiment-Assoziation anknüpft, erläutert. Es bleibt also für die Hörer unklar, ob sich das wichtig auf jene Person, Cassis' eigene Rolle oder das Verhältnis zwischen ihnen bezieht. Sie hätte, um dem Detaillierungszwang ausreichend zu genügen, hier weiter explizieren muissen.

Statt dessen kehrt sie zurück in die Traumerzählung, jetzt jedoch nicht mehr eingebettet in den argumentativen Super-Frame und damit $n$ icht mehr Teil ihres Propositional-Frame, sondern nur noch des Macro-Frames. Sie benutzt diesen Frame-Wechsel, um dem Detaillierungszwang zu entgehen. Der Focus-ChangeMarker Aber in Zeile 23 erhält hier also die Qualität eines Strategic-Move. Seine semantische Verlängerung erfährt der Strategic-Move in ihrer Aussage, daß sie sich eigentiich nur an die Farbe des Frosches erinnern kann (Zeile 24 u. 26). Zusammenfassend läßt sich hinsichtlich der semantischen Verkürzung feststellen, daß sie auf dem Local-Level dem Detaillierungszwang entgeht; auf dem Abstract-Level läßt sie die rückbeziehende Kategorie der Schlußfolgerung unausgefüllt.

Vor diesem Interpretationshintergrund läßt sich eine Hypothese bezüglich ihrer Behauptung in Zeile 39-42 aufstellen. Die Außerung, daß sie nicht an "psychologischem Auseinanderklambüsern" interessiert sei, könnte als Abwehr von Hörerrückfragen auf ihren zuerst aktualisierten Propositional-Frame angesehen werden. Eindeutig wäre dies höchstens durch späteres Befragen von Cassis zu erhärten, weshalb ich sie zu einem Gespräch bat. Dabei konfrontierte ich sie zunächst mit einem längeren Ausschnitt des Gesprächs, in den die hier analysierte Sequenz eingebettet ist. Anschließend befragte ich sie zu ihren Außerungen. 15)

Cassis bestätigte in drei wichtigen Punkten die hier entwickelte Interpretation:

1. Cassis äußerte, daß ihr Traumfragment und ihre Experiment-Assoziation zusammengehören. 
2. Sie konnte zwar auch nicht die Lücke in Zeile 10 füllen, doch erinnerte sie sich, hier auf die Störung durch Moet's Lachen abwehrend reagiert zu haben.

3. Sie beurteilte den Abbruch der narrativen Passage 2 als ein Handeln, das von der bedrückenden Erinnerung an ihre Interpretation zu dem für sie positiven Aspekt der Farbe - grün sei ihre Lieblingsfarbe - überleiten sollte. Ihre Aussage bezüglich des Desinteresses an "psychologischem Auseinanderklambüsern" charakterisierte sie selbst als unwahr, wodurch die oben aufgestellte Hypothese zwar nicht hinreichend belegt, jedoch annähernd plausibel wird.

Die frame-theoretische Interpretation führte also zu Resultaten, die von der Erzählerin weitgehend bestätigt wurden. Frame-Wechsel können als StrategicMoves verwendet werden, um semantische Verkürzungen entgegen dem Detaillierungszwang zu retten. Von Relevanz wäre nun die Frage, weshalb die Hörenden nicht intervenierten. Dabei könnte vermutet werden, daß es dem besonderen Charakter einer Traumerzählungsgruppe entspricht, auf die Anwendung von Strategien zum Schutz der eigenen Intimsphäre durch den Sprechenden mit der Befolgung einer Maxime der Akzeptanz zu reagieren. Dieser Aspekt soll jedoch hier nicht mehr behandelt werden.

Anmerkungen

1) Zur Unterscheidung zwischen Zielen und Zwecken von Kommunikation siehe Ungeheuer (1987c, 315).

2) Als Urheber dieser Richtung kann für die Kognitionspsychologie 0tto Selz gelten, dessen Arbeiten aus den ersten Jahrzehnten dieses Jahrhunderts jedoch nur selten gewürdigt werden. Beachtung aus semiotischer Sicht findet das Werk Selz' bei Hanke (1987).

3) Vgl. die klassische Definition von "Frame" bei Minsky (1975) sowie die Ausführungen bei Winograd (1977). Siehe auch Goffman (1974, 10f.). Zu Erfahrungsschemata aus phänomenal-analytischer Sicht vgl. Schütz (1981).

4) Ungeheuer (1987c, 308ff.). Die Gesamtheit der Frames eines Individuums könnte als Teil der individuellen Welt theorie angesehen werden.

5) "From a more procedural viewpoint, we can look at schemas as providing a guide for structuring the processes of production and comprehension" (Winograd 1977, 74). Dennoch beziehen sich kognitions theoretische Arbeiten vornehmlich auf das Verstehen (für die Psycholinguistik vgl. das Urteil bei van Dijk/Kintsch 1983, 261). Ober Unterschiede zwischen und Oberschneidungen von Sprachverstehens- und -produktionsprozessen vgl. van Dijk/Kintsch (1983, $261 \mathrm{f}$.).

6) Mit diesem Konzept steht ein ganz anderer Ansatz in gewisser Parallele: Die Situationstheorie des Sprachtheoretikers Philipp Wegener, bereits vor mehr als 100 Jahren vorgelegt, aber bis heute nur selten beachtet. Wegener unterscheidet die "Situation der Anschauung" ("die umgebenden Verhältnisse und die Gegenwart der angeredeten Person"), die "Situation der Erinnerung" (die einer Außerung unmittelbar vorausgegangenen und noch be- 
wußt erinnerten Ereignisse) und die "Situation des Bewußtseins" (in meiner Temninologie der Gesamt-Set der Frames eines Individuums), die insgesamt die "Gesamtsituation" konstituieren (siehe Wegener 1885, 21ff.; Würdigungen Wegeners etwa bei Juchem 1984, Scherner 1979, Ungeheuer 1987c).

7) Die Darlegungen in den verschiedenen Arbeiten van Dijks (1977a, 1977b, 1980, 1934 sowie van Dijk/Kintsch 1983) sind für das hier vorgeschlagene Modell Orientierung gewesen, obwohl terninologische Differenzen bestehen. Ich verwende Frame als Bezeichnung für geordnete kognitive Strukturen auf allen Ebenen (entgegen van Dijks Bedenken, siehe ders. 1977b, 19), versehe den Ausdruck allerdings mit einem Index. Meine Entscheidung begründe ich damit, daß alles Handeln erfahrungsbasiert ist, Erfahrungen jedoch schematisch geordnet sind (vgl. etwa Schütz 1981). Van Dijks Unterscheidung zwischen "macro-structures" und "frames" sieht letztere als konventionalisiert an, "macro-structures" als ad-hoc-Information im Gefüge des je besonderen Gesprächs. Da sie jedoch als Deutungsschema ta bzw. Interpretationsrahmen im weiteren Gesprächsverlauf dienen, verwende ich den Ausdruck Macro-Frame.

8) Vgl. "topic change marker" bei van Dijk/Kintsch (1983, passim). Zu "Fokussierung" bzw. Aufmerksamkeitssteuerung vgl. Kallmeyer (1978) sowie Bublitz/ Kühn (1981).

9) Argumentationsschemata finden sich in AnschluB an Toulmin bei Ungeheuer (1987b, 96ff.) sowie bei van 0ijk (1980). Narrative Schemata legten u.a. Rumelhart (1975) sowie van Dijk (1980) vor. Vgl. auch Kallmeyer/Schütze (1977, 176ff.). Ich siedele solche Super-Frames deshalb auf einem abstract level an, weil ihre Denotate nur Relationen zwischen Topical-, Macro- und Propositional-Frames sind. Vgl. van Dijk/Kintsch $(1983,189)$.

10) Für eine umfassende Behandlung mit dem Schwerpunkt auf Strategien des Verstehens siehe van Dijk/Kintsch (1983). Eine Anwendung des Strategiebegriffs auf die Erforschung von Vorurteilen in Gesprächen über soziale Minderheiten bei van Dijk (1984, bes. 115ff.). Vgl. auch den Beitrag von König, in diesem Band.

11) Auf diese Situation wäre unter Umständen der Begriff des "Konmunikationsrisikos" übertragbar. Vgl. Niehüser (1986).

12) Näheres über das Forschungsvorhaben und erste Zwischenergebnisse in dem "Bericht aus einer kommunikationswissenschaftlichen Werkstatt: Gespräche über Träume" mit verschiedenen Beiträgen in SPIEL 7 (1988), H.1. Zum Konzept der Untersuchung siehe besonders den Beitrag von Goetze/Hanke/Richter (1988).

13) Siehe zu den in der Untersuchung verwendeten Transkriptionsverfahren und zu Transkription aligemein Wolf (1988).

14) Zu "Unschärfeindikatoren" bzw. "Hedges" vgl. Müller (1980) und (1984) sowie Lakoff (1972).

15) Zum Verfahren der Konfrontation der Versuchsperson mit ihrer eigenen Aufzeichnung siehe Kalbermatten/von Cranach (1981).

\section{Literatur}

Bublitz, W./Kühn, P. (1981), Aufmerksamkeitssteuerung: Zur Verstehenssicherung des Gemeinten und des Mitgemeinten. In: Zeitschrift für germanistische Linguistik 9, 55-76. 
van Dijk, T. (1977a), Context und Cognition: Knowledge Frames and Speech Act Comprehension. In: Journal of Pragmatics 1, 211-231.

van Dijk, T. (1977b), Senantic Macro-Structures and Knowledge Frames. In: Just/Carpenter (1977), 3-32.

van Dijk, T. (1980), Textwissenschaft. Eine interdisziplinäre Einführung, München ( $d t$. übers.).

van Dijk, T. (1984), Prejudice in Discourse. An Analys is of Ethnic Prejudice in Cognition and Conversation, Ams terdam.

van Dijk, T./Kintsch, W. (1983), Strategies of Discourse Comprehension, New York/London usw.

Goetze, J. (1988), Ober den alltäglichen Gebrauch von Traumerzählungen in psychoanalytischer Sicht. In: SPIEL 7, H. 1.

Goetze, J./Hanke, M./Richter, H. (1988), Traumerzählungen und Traumberichte in Alltagskommunikation. In: SPIEL 7, H. 1.

Goffman, E. (1974), Frame Analysis. An Essay on the Organisation of Experience, New York/Evanston usw.

Hanke, M. (1987), Zur Psychologie kreativer Semiosis: Otto Selz. Vortrag auf den Kongreß für Semiotik in Essen 1987 (Ms.).

Hanke, M. (1988), Träumer, Traum und Adressat - Traumdarstellungen in Alltagskommunikation. In: SPIEL 7, H. 1.

Hanke, M./Schmitz, M. (1988), Kommunikation über Träume im Alltag. Zur Auswertung eines Fragebogens. In: SPIEL 7, H. 1.

Juchem, J.G. (1984), Die Konstruktion des Sprechens. Kormunikationssemantische Betrachtungen zu Philipp Wegener. In: Zeitschrift für Sprachwissenschaft 3, 3-18.

Just, M.A./Carpenter, P.A. (1977), (Hrsg.), Cognitive Processes in Comprehension, Hillsdale/New Jersey.

Kalbematten, U./von Cranach, M. (1981), Hierarchisch aufgebaute Beobachtungssysteme zur Handlungsanalyse. In: Winkler, P. (Hrsg.), Methoden der Analyse von Face-to-Face-Situationen, Stuttgart, 83-127.

Kallmeyer, W. (1978), Fokuswechsel und Fokussierungen als Aktivitäten der Gesprächskonstitution. In: Meyer-Hermann, R. (Hrsg.), Sprechen - Handeln Interaktion. Ergebnisse aus Bielefelder Forschungsprojekten zu Texttheorie, Sprechakttheorie und Konversationsanalyse, Tübingen, 191-241.

Kallmeyer, W./Schuitze, F. (1977), Zur Konstitution von Kommunikationsschenata der Sachverhal tsdarstellung. In: Wegner, D. (Hrsg.), Gesprächsanalysen. Vorträge, gehalten anläßlich des 5 . Kolloquiums des Instituts für Kommunikationsforschung und Phonetik Bonn, 14.-16. Okt. 1976, Hamburg, 159-274.

Lakoff, G. (1972), Hedges: A Study in Meaning Criteria and the Logic of Fuzzy Concepts. In: Hockney, D./Harper, W./Freed, 8. (Hrsg.), Contemporary Research in Philosophical Logic and Linguistic Semantics. Proceedings of a Conference Held at the University of Western Ontario, Canada, Boston, 221-271.

Minsky, M. (1975), A Framework for Representing Knowledge. In: Winston, P.H. (Hrsg.), The Psychology of Computer Vision, New York, 211-280.

Miiler, K. (1980), Interaktionssemantik. In: Deutsche Sprache 8, 289-305.

Muiller, K. (1984), Rahmenanalyse des Dialogs. Aspekte des Sprachverstehens in Alltagssituationen, Tübingen. 
Niehüser, W. (1986), Vermeidung kommunikativer Risiken. In: Hundsnurscher, F./Weigand, E. (Hrsg.), Dialoganalyse. Referate der 1. Arbeitstagung Münster 1986, Tübingen 213-224.

Rumelhart, D.E. (1975), Notes on a Schema for Stories. In: Bobrow, D.G./Collins, A. (Hrsg.), Representation and Understanding. Studies in Cognitive Science, New York/San Franzisco etc., 211-236.

Scherner, M. (1979), Nichttextualisierte Verstehensvoraussetzungen als sprachwissenschaftliches Problem. In: Bülow, E./Schmitter, P. (Hrsg.), Integrale Linguistik. Festschrift für Helmut Gipper, Amsterdam, 319-357.

Schïtz, A. (1967), On Multiple Realities. In: ders., Collected Papers I. The Problen of Social Reality, The Hague, 207-259.

Schiitz, A. (1981), Der sinnhafte Aufbau der sozialen Welt. Eine Einleitung in die verstehende Soziologie, Frankfurt/M. (Nachdruck. 1932).

Selz, I. (1981), Die Gesetze der produktiven und reproduktiven Geistestätigkeit. Kurzgefaßte Darstellung. In: Groffmann, K.J. (Hrsg.), Leben und Werk von 0tto Selz (1881-1943). Zum 100. Geburtstag des Philosophen und Psychologen, Mannheim, 31-61 (Nachdr. v. 1924).

Ungeheuer, G. (1987a), Kommunikations theoretische Schriften I: Sprechen, Mitteilen, Verstehen (hrsg. von J.G. Juchem), Aachen.

Ungeheuer, G. (1987b), Kommunikationssemantik: Skizze eines Problemfeldes. In: ders. (1987a), 70-99.

Ungeheuer, G. (1987c), Vor-Urteile über Sprechen, Mitteilen, Verstehen. In: ders. (1987a), 290-338.

Wegener, Ph. (1885), Untersuchungen über die Grundfragen des Sprachlebens, Halle.

Winograd, T. (1977), A Framework for Understanding Discourse. In: Just/Carpenter (1977), 63-88.

Wolf, G. (1988), Reflexionen und Erfahrungen: Ober "Transkription" als Instrument und Quelle von Kommunikationsforschung. In: SPIEL 7, H.1. 
Anhang

c [(Und) bel alr let das Immer n/sehr abhängls dann auch v/ähn/ja

c [auch von dom Traur m/inwlewelt da irsendwas vecbels 1s. He

c $[($ sochluckt $))$ manchaal. werden sachen auch erst ih wichtis lrgend-

c [welche symbole oder Irsendwas in nem Traum durch die Farbe.

5 C Jetze hab lch auch vor 'n panr tagen uns geträumt von elnea sehr

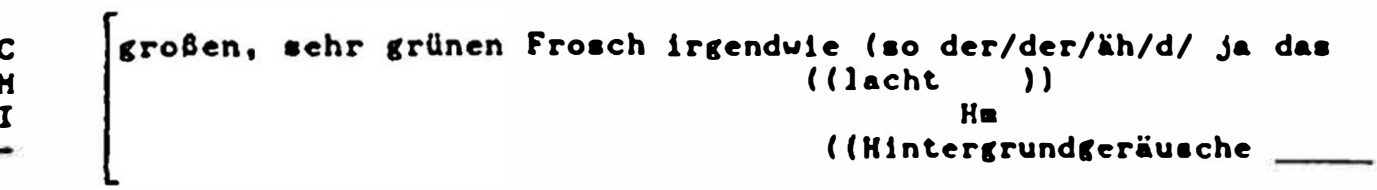

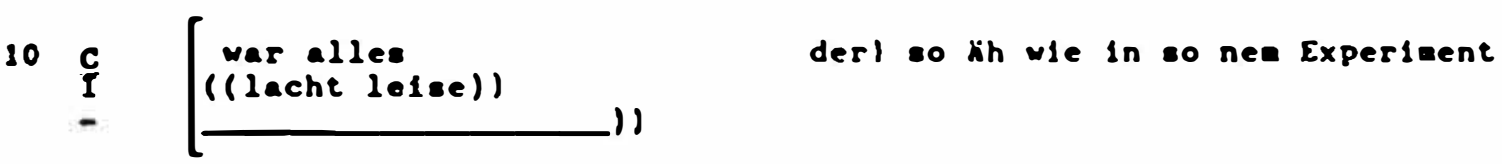

C [lar was wols lch, wenn man erforsche, wle/wle Muskelzuckungen

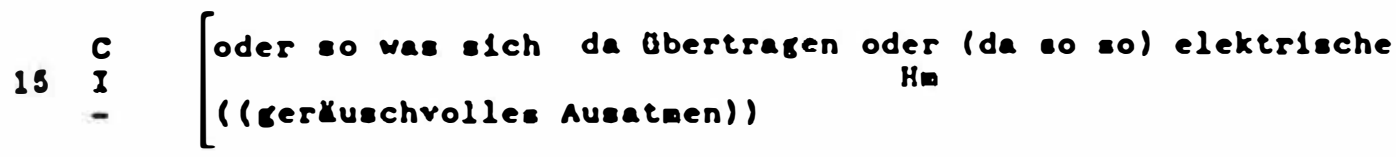

C [strbne da durch dle Muskeln Behen oder was, Irsendwo ausgehingt

C [war, (ganz) surchterllch. Mlr 1. dann auch 1a/la Nachhlneln dann

C [aupgefa/aleo slosefallen, wt was es zu tun hatte (hinterher),

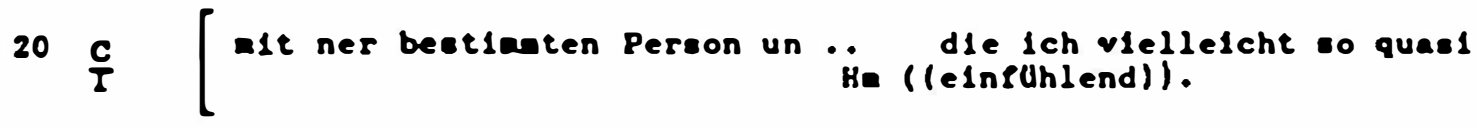

C [so hab $1 \mathrm{ch}$ als das dann cedeutet auch so als $\mathrm{kh}$, ha. al. Ver-

C [uchskaninchen halt sebrauche .. Aber der war sehr zean, dleser

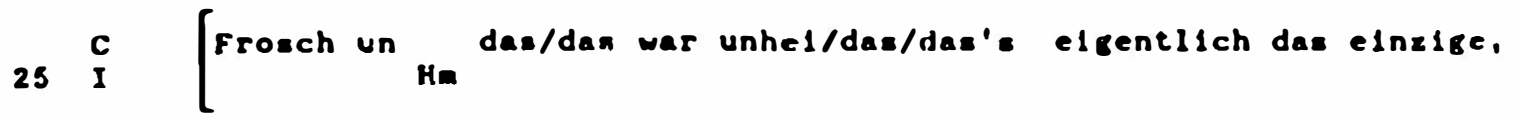

c [was lch alch noch erinnern kann, was lch nlch aurseschrleben hab.

C [Ich hatte auch schon Trlune/ also bel alr in den Traun is das 
c [Lesl unterschiedlich eben, annchanl ind' riehtise Geschich-

c [ten so, manchael aind's nur solche Fetzen, mancheal 1. es nur

30 C [dles/dielso ne/1rn/so ne Farbe oder. « $\ldots .$.

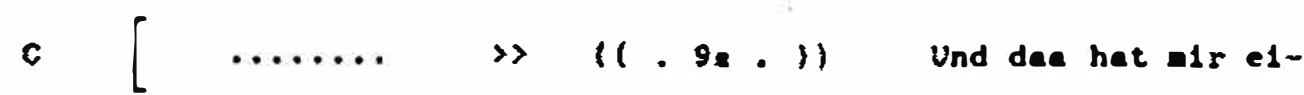

C [rentlich auch lust de draus remacht mal zu hören, wle es bel den

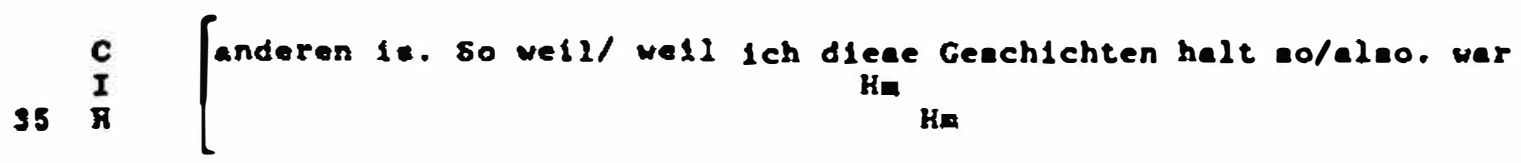

C [auch der Ansporn $n / 1 n$ die Gruppo halt relnzugehen oder alt den

C [anderen denn die Gruppe enzutangen

C [Nich Jetzt veil/well $1 \mathrm{ch}$ also da. in das Inseresee hatte irgend-

10 C $\quad[$ wle voleo. parchologlech das Jetz auseinanderzuklambisern, son-

c [ders eher so die Neusler aut die Geschichien halt. $11,11.11$

Erläuterung der Transkriptionszeichen:

(Und) : fast sicher identifiziertes Wort

( ) : leere $\mathrm{Klammer}=$ unidentifizierbare Kußerung

(1) : paralinguistische Signale b2w. Kommentare des Transkribenten

/ : markiert Stottem, Unterbrechung $0 . \ddot{a}$.

total : Betonung

- $\quad$ : Satzende oder kurze Pause
.. $\quad$ : ca. zweisekündige Pause

. 95. : 9 Sekunden Pause (in Doppelklammern)

Die Winkelklammern markieren eine Auslassung im Transkript. 


\section{DuEPublico}

Dieser Text wird über DuEPublico, dem Dokumenten- und Publikationsserver der Universität Duisburg-Essen, zur Verfügung gestellt. Die hier veröffentlichte Version der EPublikation kann von einer eventuell ebenfalls veröffentlichten Verlagsversion abweichen.

DOI: $\quad 10.1515 / 9783111566672.323$

URN: urn:nbn:de:hbz:464-20210111-132219-7

Wolf, Guido: Über semantische Verkürzungen in Alltagsgesprächen. Zur Applikation eines rahmentheoretischen Konzepts auf empirisches Material.

In: Dialoganalyse II : Referate der 2. Arbeitstagung, Bochum 1988. Band $2 /$ Herausgegeben von Edda Weigand, Franz Hundsnurscher. Tübingen: Max Niemeyer Verlag, 1989. ISBN 978-3-484-30230-3 (print) - Reprint: Berlin: De Gruyter, 2010. ISBN 978-3-11-156667-2 (PDF). DOI: https://doi.org/10.1515/9783111566672. S. 323 - 336

(c) 2010 Walter de Gruyter Alle Rechte vorbehalten. 